\title{
Análisis temático sobre la fibromialgia en la prensa escrita Argentina: descripciones generales y el rol de la psicología
}

Mercedes Sarudiansky(a)

Sarudiansky M. A thematic analysis on papers about fibromyalgia in the Argentine printed press: general descriptions and the role of psychology. Interface (Botucatu). 2016; 20(56):25-36.

Since fibromyalgia was first conceptualized in 1990, it has been studied in terms of its validity as a diagnosis, its definition as a disease and its treatment. These issues have been addressed within different biomedical and social sciences disciplines, including psychology, psychiatry, anthropology and sociology. In this paper, we discuss how knowledge about fibromyalgia is disseminated in Argentina, through thematic analysis of the papers on this syndrome published in the printed press. We analyze how newspaper articles describe fibromyalgia - including general characteristics, causal hypotheses and treatments - and how the media describes the role of psychology in its treatment.

Keywords: Fibromyalgia. Means of communication. Thematic analysis. Psychology.
Desde su conceptualización en 1990, la Fibromialgia (FM) ha sido discutida en términos de su validez como diagnóstico, su definición como enfermedad y sus tratamientos. Estas cuestiones han sido abordadas por diferentes disciplinas biomédicas y sociales, entre las cuales se incluyen la psicología, la psiquiatría, la antropología y la sociología. En este trabajo, indagaremos cómo se difunden los saberes respecto de la FM en Argentina, a través de un análisis temático de los artículos referentes a este síndrome publicados en la prensa escrita. Analizaremos la manera en que las notas periodísticas describen a la FM - características generales, hipótesis causales, y tratamientos $-\mathrm{y}$ reflexionaremos respecto de cómo estos medios abordan el rol de la psicología en el tratamiento de esta dolencia.

Palabras clave: Fibromialgia. Medios de comunicación. Análisis temático. Psicología. 


\section{Introducción}

Dentro del ámbito médico, se conoce como Fibromialgia o Síndrome de Fibromialgia (de ahora en más, "FM") a un cuadro caracterizado por dolor generalizado en distintos lugares del cuerpo, asociado con alteraciones en el sueño, fatiga y emocionales, como depresión y ansiedad ${ }^{1,2}$. Pese a ser un diagnóstico controvertido ${ }^{2,3}$ y no totalmente aceptado por la comunidad profesional, ha sido incluido dentro de la nomenclatura de la Clasificación Internacional de las Enfermedades de la Organización Mundial de la Salud, bajo el código M79.04.

Aunque se trata de un diagnóstico relativamente novedoso - la primera sistematización oficial de sus síntomas datan de 19901, pueden hallarse descripciones de este tipo de enfermedades desde hace más de 500 años, siendo la fibrositis - propuesto por Gowers en 1904 - su antecedente nosológico más directo 5 .

Pese a ser considerado un síndrome reumatológico, es tema de interés de la psicología, dado el énfasis que se ha puesto a los aspectos psicológicos que intervienen en los procesos de percepción del dolor a partir de la "teoría de puerta" de Melzack y Wall ${ }^{6}$. Estos autores explican que la percepción del dolor no es proporcional al daño recibido, sino que existen mecanismos corticales que influyen en la manera en que las personas experimentan el dolor, como emociones y pensamientos.

Desde entonces se abrió un campo desde las ciencias psicológicas sobre el dolor, entre las cuales se incluyen aportes del psicoanálisis - p.e., procesos inconscientes en los cuadros conversivos ${ }^{7}-$ neuropsicológicos y cognitivos - p.e. procesos de procesamiento de la información y de funciones cognitivas básicas ${ }^{8}$, de terapia cognitivo-conductual9-11, entre otras.

En la actualidad, la FM es un síndrome que se encuentra incorporado dentro de los ámbitos profesionales y de la población general, lo cual se refleja en un número creciente de publicaciones en revistas y presentaciones en congresos científicos, como también las charlas abiertas a la comunidad, y la divulgación de los conocimientos al público general, a través de medios masivos de comunicación.

Los medios de comunicación escrita son una interesante fuente de conocimiento, dado que reflejan aquella información que se difunde entre la población, y que tiene alcance a un segmento extenso de personas. A su vez, en lo que hace al conocimiento científico, da cuenta de los sesgos a partir de los cuales esta información se difunde - de lo "real e importante"12, ya que sólo una pequeña parte de las investigaciones se refleja en los medios masivos. De todas maneras, tal como plantea Duarte Rodrigues $^{13}$, es menester destacar que, dada la naturaleza vicaria del campo mediático, es esperable encontrar características específicas que lo diferencien de los discursos propios del campo médico especializado, aspecto que intentaremos explicitar en la Discusión, relacionando los resultados del presente análisis y la información en la literatura científica.

Asimismo, la cobertura periodística ocupa un rol importante respecto de la comprensión, el juicio de valor y las actitudes del público en general sobre temas relacionados con ciencia y tecnología $a^{14-16}$. Las creencias de la población general sobre la salud y la enfermedad han sido un tema de interés para el campo de las investigaciones en salud, dado que las representaciones sociales ${ }^{17}$ sobre la aflicción y la cura impactan sobre la manera enque las personas se comportan ante experiencias de enfermedad ${ }^{18-21}$. Teniendo en cuenta que los diarios y revistas de difusión masiva son una fuente importante de información consultada por el público en general -y que pueden influir en los preconceptos de la población sobre los temas de salud ${ }^{22}$, consideramos esencial indagar sobre estas cuestiones para registrar las creencias de las personas en relación a temas de salud. El caso de la FM es en particular interesante - como también el autismo ${ }^{23}$ o la sexualidad ${ }^{24}$ - dado el carácter controvertido del diagnóstico, que podría caracterizarse como un cuadro "ambiguo" ${ }^{19}$, que se presentan más permeables a los efectos de la cultura y el contexto.

En esta ocasión, indagamos la difusión de saberes sobre FM en la prensa escrita argentina. Comenzaremos describiendo la presencia de la FM en estos medios. Luego analizaremos cómo las notas periodísticas la describen y caracterizan. Finalizaremos reflexionando respecto del lugar que ocupa la psicología en la conceptualización y las intervenciones propuestas que se difunden en estos medios. Este artículo forma parte de una investigación más amplia sobre la FM en el contexto de la Ciudad Autónoma de Buenos Aires, Argentina, y alrededores. 


\footnotetext{
(b) La Nación tiene un perfil conservador vinculado a instituciones tradicionales, como la Iglesia Católica, el sector empresarial y las fuerzas armadas; Clarín se autodefine progresista e independiente. Ambos se encuentran en explícita confrontación con el gobierno actual. Página/12 se dirige a los sectores medios intelectuales, mientras que Diario Popular se dirige a los sectores populares. (Fuente: IVC. Disponible en: http://sinca.cultura. gov.ar/sic/estadisticas/i. php?id $=130 \& g r$ type $=$ column).
}

\section{Metodología}

Se realizó un análisis temático y de contenido de los artículos sobre FM obtenidos a partir de las páginas web de cuatro diarios argentinos de mayor tirada - La Nación, Clarín, Página/12 y Diario Popular(b). La búsqueda fue realizada a partir de un solo descriptor ("Fibromialgia"), desde 1999 (cuando aparece el primer artículo) hasta julio de 2014. Los registros también incluyen artículos de revistas asociadas a estos diarios, que aparecen en los resultados de los buscadores.

Los pasos para el análisis temático se basaron en la guía propuesta por Braun y Clarke 25 ; a partir de la lectura y relectura del material, codificamos los aspectos más relevantes de los datos, agrupando estos códigos en temas más amplios que permitan una descripción profunda y detallada del fenómeno.

Primeramente, organizamos el material a partir de categorías propias y basadas en trabajos similares del contexto argentino ${ }^{26,27}$, entre las cuales incluimos: Diario en el que fue publicado; tipo de artículo (informativos, anuncios o columnas editoriales); sección en la que fue publicado; tema principal (cuyas categorías serán detalladas en el próximo punto); si cuentan con testimonios de profesionales o especialistas en el tema; y si cuentan con datos de investigaciones locales o internacionales. Estos datos fueron volcados en una matriz para facilitar su sistematización y análisis. La información fue analizada cuantitativamente, permitiéndonos esbozar un panorama general de la presencia de la FM en los medios gráficos.

Luego realizamos un análisis temático propiamente dicho del contenido de los artículos. Enfocamos nuestro análisis sobre aquellos artículos que hagan una descripción del fenómeno $(n=16)$, y no solamente una mención. Seleccionamos los primeros códigos, que agrupamos en siete temas: (a) definiciones de la FM - palabras que los pacientes o especialistas utilizan para nombrarla, (b) causas explicaciones sobre etiología, (c) tratamientos -abordajes terapéuticos asociados a la FM, (d) diagnóstico - qué criterios se utilizan para diagnosticar la FM, (e) síntomas - qué sintomatología caracteriza al paciente con FM, (f) prevalencia referencias a la población con FM y el porcentaje de casos en población general, y (g) características de los pacientes con FM - cómo aparecen caracterizadas las personas con FM.

Finalmente, para dar cuenta del lugar que ocupa la psicología en la difusión de la FM, analizamos el material a partir de distintas palabras clave que impliquen temas "psi" -"psicología", "psicólogo/a(s)", "psiquiatría", "psicógeno", "psicosocial", y otras palabras clave relacionadas, como "estrés", "ansiedad", "depresión", "emoción", "cognición", y sus derivados. Diferenciamos aquellos artículos en los que estas palabras estaban presentes $(n=29)$ y en los que no $(n=37)$. Luego, clasificamos la manera en que se incluye a estos aspectos "psi" en cinco categorías, que describiremos en los Resultados.

\section{Resultados}

\section{Sobre la difusión de la FM en la prensa escrita argentina}

Hemos relevado un total de 66 referencias sobre la FM en los registros digitales de cuatro diarios argentinos. Encontramos registros desde el año 1999, que demuestran una presencia creciente de este tema. Agrupando los resultados en períodos de cinco años, desde el primer registro (1999-2004) encontramos 
sólo siete referencias; en el período de 2005-2009 éstas ascienden a 24, llegando a 36 registros en los últimos cinco años (2010-julio 2014) (Figura 1).

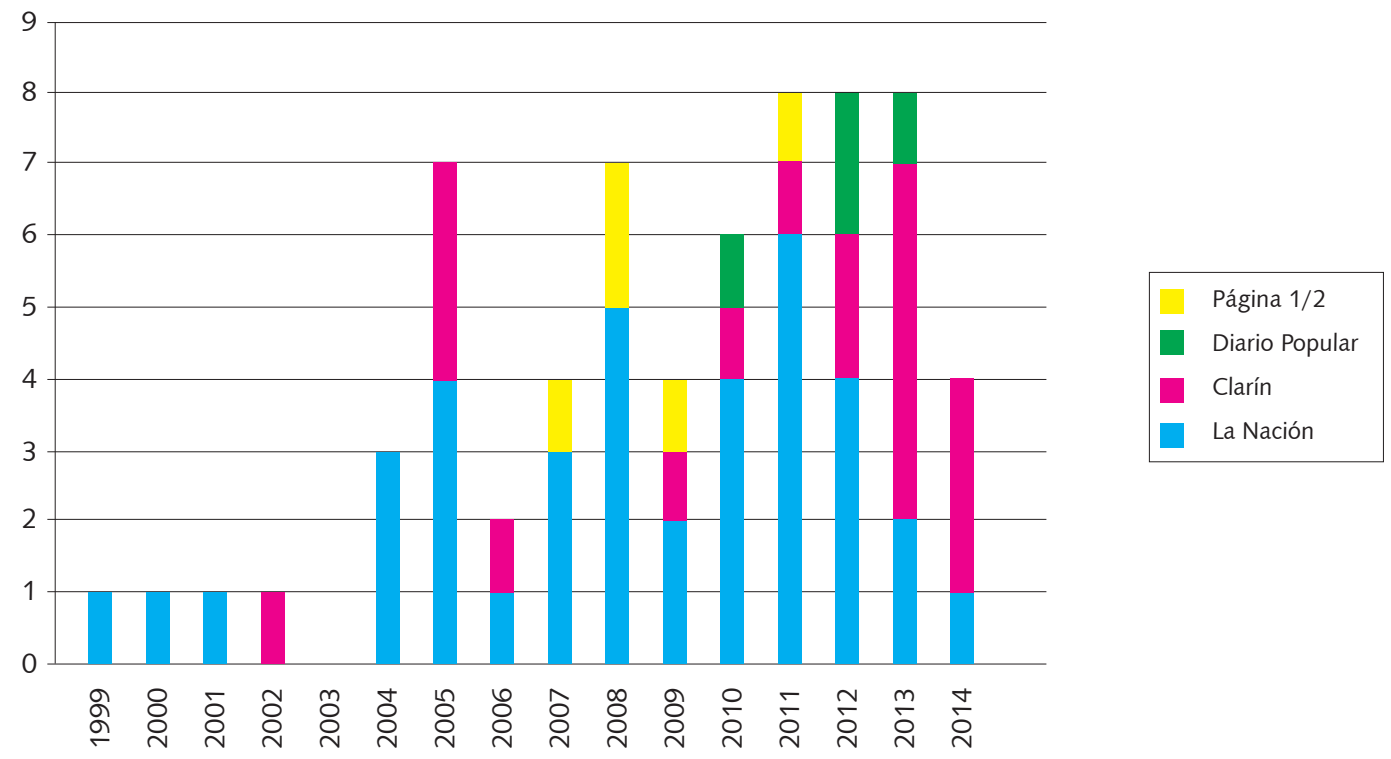

Figura 1. Publicaciones sobre fibromialgia por año (1999-2014)

La mayoría de los artículos $(n=30)$ se agrupan en secciones que tratan temas sobre ciencia y salud; 12 en las secciones sobre temáticas referidas a la sociedad, mientras que el resto se distribuye en secciones diversas, como arquitectura, moda y belleza, revistas e información general.

De estos 66 registros, la mayoría $(n=58)$ son notas de carácter informativo - en las que se informa a la población respecto de avances o noticias científicas, cuatro corresponden a anuncios - publicaciones en los que se promocionan eventos, mientras que el resto $(n=4)$ son cartas de lectores o columnas editoriales.

Del total de los artículos, sólo seis registros indican la palabra "fibromialgia" en el título. Sin embargo, agrupando los artículos en función de su contenido temático, son 16 los registros que ubican a la FM como tema principal. El resto de los artículos fueron clasificados de la siguiente manera: Dolor $(n=6)$, Enfermedades reumáticas $(n=5)$, Otras patologías $(n=12)$, Terapias $(n=14)$, Otros temas relacionados con la salud $(n=13)$, Otros temas no relacionados con la salud $(n=1)$. La FM como tema en sí misma no se desarrolla en todos los casos, sino que en muchas ocasiones sólo se nombra dentro de temas heterogéneos. Esto puede deberse a que la FM es una enfermedad de reciente conceptualización, y, hasta que se instaló como un tema en sí mismo, era tratada como un aspecto secundario en artículos de temáticas diversas. En la sección siguiente haremos énfasis en aquellos artículos en los cuales se describe a la FM, para dar cuenta de qué tipo de información es la que circula en los medios locales sobre este cuadro. 
Otro dato de interés es que, pese a que la mayoría cuentan con los testimonios de reconocidos especialistas en el tema de distintas disciplinas $(n=43)$, son muy pocos los que hacen referencia a investigaciones o datos locales $(n=8)$. La mayor parte de estos artículos $(n=26)$ citan investigaciones de instituciones de EEUU o Europa. Esto puede deberse, por un lado, a que las investigaciones en estas temáticas no han ganado suficiente espacio a nivel local, o bien que es necesaria una mayor difusión pública de los avances en investigación sobre este tema. Desarrollaremos este tema en el apartado Discusión.

\section{La descripción de la FM}

Del total de los 66 artículos, 16 realizan una descripción sobre las características de la FM. De estos artículos identificamos siete categorías para caracterizar los aspectos de la FM que circulan en los medios de comunicación gráfica.

\section{a. Definiciones de la FM}

El contenido de la información dentro de esta categoría parte de la pregunta general "¿qué es la FM?". Las respuestas que se desprenden de los 16 artículos las subdividimos en cinco subcategorías:

- Inespecificidad: En muchas ocasiones $(n=9)$ la FM no era presentada como una enfermedad definida, sino de maneras inespecíficas, tales como "malestar generalizado", "un mal", o "una carga". También describen que es difícil de diagnosticar, que sus síntomas pueden confundirse con otras enfermedades, y que afecta a diferentes áreas de la vida del paciente.

- Especialidad/Localización: Pese a este carácter inespecífico, varios artículos $(n=5)$ también la definen en virtud de la especialidad médica que la estudia ("enfermedad reumatológica"), o bien de su presunta localización ("enfermedad del sistema nervioso", "enfermedad músculo-esquelética", "psicoinmunoneuroendócrina").

- Duración: Todos los artículos destacan el carácter crónico de la FM, lo cual se asocia al hecho concreto de ser un malestar de larga duración, y a la falta de estrategias terapéuticas efectivas ("no tiene cura").

- Funcionalidad: La mayoría $(n=14)$ señalan que la $F M$, pese a no ser una enfermedad grave, afecta a la funcionalidad del paciente.

- Dolor: Una de las características destacadas de la FM es la presencia de dolor, siendo muchas veces $(n=14)$ definida a partir de éste ("Dolor crónico extendido", "Mayor sensibilidad al dolor", "Respuesta anormal al dolor"). Asimismo, el dolor puede referirse de manera inespecífica -"dolor generalizado"-, a zonas específicas - "cervicales, cuello y espalda", a los "puntos gatillo" definidos por el Colegio Norteamericano de Reumatología (ACR)1-, o a síntomas asociados, como cefaleas o migrañas.

\section{b. Causas}

Once artículos hacen referencia a la etiología de la FM. En la mayoría de ellos, se hace referencia a una sola causa - "desconocida", "neurológica", por "estrés" o "sobrecarga", mientras que un solo artículo refleja a la FM como determinada por múltiples causas, entre ellas ambientales - conflictos laborales, familiares, infecciones o accidentes, biológicas - desarreglos hormonales, inmunológicos y neurológicos, o como un desequilibrio o desarmonía general.

El desencadenante puede ser tan diverso como una infección viral o bacteriana, algún conflicto familiar o laboral, un divorcio, traumatismos (golpes, caídas), un accidente automovilístico o estrés posquirúrgico [...] Las evidencias científicas están aportando datos sobre el estrés crónico, que provoca la desadaptación del eje del cortisol, la hormona del estrés. ${ }^{28}$

Estas características coinciden con el carácter inespecífico a la hora de plantear una definición de la FM. 


\section{c. Tratamientos}

Catorce artículos hacen referencia a tratamientos indicados o utilizados por pacientes. La gran mayoría de ellos hace énfasis en los abordajes medicamentosos - analgésicos, antidepresivos, anticonvulsivantes (principalmente Pregabalina). También se mencionan los abordajes psicoterapéuticos y educativos, programas de actividad y terapia física, y opciones de medicina complementaria/ alternativa.

Pese a contadas excepciones - en las que sólo se hace mención de las opciones medicamentosas, la mayoría de los artículos coinciden en que es la complementariedad terapéutica, más que la monoterapia, el modelo de abordaje más recomendado.

\section{d. Diagnóstico}

Con respecto a los criterios diagnósticos, sólo dos artículos hacen referencia a aquellos establecidos por el $\mathrm{ACR}^{1}$, el único disponible hasta el momento. Asimismo, los artículos que hacen mención de los métodos o criterios diagnósticos se enfocan en la aplicación de técnicas de neuroimagen -resonancia magnética o electroencefalograma, y en la evaluación clínica. A su vez, destacan que, dado que en la mayoría de los casos los test biológicos resultan normales, se dificulta el diagnóstico y conlleva a que éste sea tardío, y atenta respecto de la credibilidad de los pacientes.

\section{e. Síntomas}

Tal como señalamos anteriormente, una de las maneras de definir a la FM implica la presencia de dolor, ubicándose como el síntoma principal. Sin embargo, en la mayoría de los artículos encontramos que la FM conlleva, además, síntomas diversos que acompañan la experiencia de dolor, y que éste puede localizarse en distintas zonas del cuerpo - que varían según el artículo, aunque suelen circunscribirse a las zonas del cuello, la espalda y las articulaciones, o bien ser un síntoma asociado, como es el caso de las cefaleas. Otros síntomas asociados a la FM incluyen: Síntomas físicos, como anquilosamiento, parestesias, problemas génito-urinarios, calambres, tensión muscular, palpitaciones, mareos, trastornos del sueño, rigidez, bruxismo; síntomas psicológicos, como cambios de humor, depresión, ansiedad, conductas impulsivas, dificultades para tomar decisiones, trastornos en la memoria, dificultades en la concentración. Asimismo, suelen asociarse con otros síndromes o enfermedades tales como: Síndrome de fatiga crónica, Síndrome del Intestino Irritable o Síndrome del Túnel Carpiano. En cuanto a estos últimos, en general no se especifica si éstos se contemplan como entidades mórbidas en sí mismas -comorbilidades-, o en virtud de los síntomas que las caracterizan.

\section{f. Prevalencia}

En cuanto a la prevalencia, la mayoría de los artículos que mencionan estas características utilizan datos de encuestas realizadas en países extranjeros, y refieren que ésta se estima entre un 2 y un $4 \%$ de la población general de adultos. Asimismo, enfatizan que es más común entre mujeres -" 20 a 1", o "80-90\%" -de más de 30 años, o de "mediana edad".

\section{g. Características de los padecientes}

Además de las características que nombramos en el punto anterior, el perfil de las personas con FM se asocia con "pacientes quejosos crónicos", en diferentes áreas de su vida - no sólo en lo que se refiere a la percepción del dolor, que consultan con diferentes especialistas - esto es, presentan extensos derroteros médicos, ligado a tanto a las dificultades diagnósticas que implica la FM como al carácter de los pacientes.

En el caso más extremo, se refieren a los pacientes como disfuncionales, que "no se adaptan", lo cual puede relacionarse con las características propias de la FM que describimos en apartados anteriores. 


\section{El lugar de la psicología}

Las menciones en torno a los aspectos psicológicos es variable en la totalidad de los artículos encontrados. Esto puede deberse a la diversidad de temas que involucró la búsqueda inicial - que van desde artículos específicos sobre FM o sobre enfermedades reumáticas o neurológicas, hasta artículos que refieren a temas ajenos a la salud o la ciencia. Por esta razón, también en este aspecto hemos circunscripto nuestro análisis a aquellos artículos en los que la FM aparece como tema principal o destacado.

A partir del análisis del contenido de los artículos tomando en cuenta las palabras clave referidas en la Metodología, organizamos el material en cinco temas principales, que describiremos a continuación:

\section{a. Factor}

Integran esta categoría aquellas menciones que hacen referencia a los aspectos "psi" como intervinientes en la experiencia de dolor y, por consiguiente, en la conformación del cuadro clínico de la FM. Por ejemplo: "Existe el prejuicio de que las mujeres se quejan más de dolor que los hombres y se atribuye a factores psicológicos" 29 .

Estas cuestiones enfatizan la perspectiva del dolor desde un punto de vista multidimensional, y no solamente como una experiencia sensorial unidireccional, ubicando a las variables psicológicas en un nivel de importancia similar a las fisiológicas.

\section{b. Estrategia terapéutica}

Como señalamos anteriormente, entre las intervenciones terapéuticas mencionadas en los artículos se incluyen los tratamientos psicológicos y/o psiquiátricos. Éstos muchas veces se asocian con los aspectos psicológicos que integran la dolencia, orientándose tanto hacia los factores intervinientes en el cuadro mismo, como a las comorbilidades asociadas.

Asimismo, en la mayoría de los casos, este tipo de estrategias se presentan de manera genérica, sin discriminar entre tipos de intervenciones o de corrientes teóricas de la psicoterapia, excepto un caso, en el que se nombran a la Terapia Cognitivo-Comportamental y la Terapia Interpersonal, para el tratamiento de la depresión.

\section{c. Patología}

Otra de las maneras en que las disciplinas "psi" se incluyen es a través de la vertiente patológica de los fenómenos mentales. De esta manera, se hace referencia a los "trastornos mentales" o "desórdenes psicológicos", en general, o bien a cuadros específicos que pueden ser rastreados en las nosografías psicológico-psiquiátricas, como ansiedad y depresión.

\section{d. Especialista}

Esta categoría engloba aquellas referencias que hacen alusión a los especialistas o profesionales "psi". La mayoría de las veces éstos se refieren a "especialistas" en enfermedades graves o dolencias crónicas, que dan cuenta de sus testimonios; en ningún momento se especifica, como sucede en el caso de los tratamientos, la corriente teórica del profesional en cuestión.

Asimismo, son pocas las referencias a los profesionales del ámbito de la salud mental que se ven implicados en estas temáticas, siendo la mayoría de las veces profesionales del ámbito de la biomedicina - neurólogos, anestesiólogos y reumatólogos, tanto del contexto local como del internacional, quienes aparecen como referentes del área.

\section{e. Disciplina}

En menor medida, los artículos se refieren a los aspectos "psi" como disciplina profesional, del ámbito de la salud, que se encuentra implicada en el tratamiento de estas temáticas: "También desde las neurociencias y desde la psicología, hay desacuerdo" ${ }^{30}$. 
También en este caso observamos que la referencia a la psicología se hace sin distinguir o definir las áreas de implicancia, corriente teórica o la especialidad a la que se refiere, a diferencia de lo que sucede con respecto a las disciplinas biomédicas.

\section{Discusión}

A partir de los datos recabados para el presente artículo, podemos dar cuenta de que la presencia de la FM en los medios de comunicación gráfica argentina presenta una tendencia creciente desde fines de la década del '90, que va desde artículos aislados en los primeros años, hasta un promedio de ocho artículos por año, en los últimos cinco años. Sin embargo, la aparición de este tema fue relativamente tardía si tenemos en cuenta que los criterios diagnósticos de FM como entidad mórbida datan de 1990', mientras que la primera aparición en los registros es de 1999.

En cuanto a la manera en que se representa la FM, la mayoría de las veces aparece mencionada en una enumeración de afecciones de distinto tipo, encontrándose pocos artículos (16) en los que se explique qué es la FM. Tomando en cuenta los temas de los artículos en los que se integra a la FM, en su mayoría se encuentran relacionados con cuestiones relativas a la salud, siendo las más frecuentes las enfermedades reumáticas, las ofertas terapéuticas, y el "dolor". Esto último tiene sentido, dado que una de las características más destacadas que se utiliza para definir a la FM es la experiencia de dolor por parte de los consultantes. De hecho, en la bibliografía especializada, la FM se encuentra englobada dentro de los síndromes o enfermedades de dolor crónico, siendo la presencia de sensibilidad o dolor en 11 de 18 puntos - "puntos gatillo" - una de las maneras más difundidas para realizar un diagnóstico certero ${ }^{1}$. Sin embargo, en los últimos años se comenzaron a realizar estudios para encontrar métodos diagnósticos alternativos ${ }^{2}$, y que no se asocie a la FM erróneamente con trastornos de músculos periféricos $^{31}$, o se haga caso omiso de los otros síntomas que caracterizan a la $\mathrm{FM}^{32}$. Incluso en esta nueva propuesta, el dolor continúa siendo uno de los aspectos más importantes para el diagnóstico y la definición de la FM como entidad mórbida ${ }^{3}$. De todas maneras, como ha notado Ponciano Aragon $^{33}$, en cada uno de estos intentos de sistematizar y de organizar un corpus de conocimiento que otrora parecía una "comunicación esquizofrénica" (p. 159), se pierde la comprensión global de la complejidad singular del sufrimiento, cuestión que también se refleja en los artículos recabados.

Otro aspecto interesante es que los padecientes son mayormente mujeres. Las cuestiones referidas al género han sido tenido en cuenta por algunos autores ${ }^{34}$, quienes explican a partir de factores biológicos - mutaciones genéticas, alteraciones en mecanismos sensoriales, psicológicos - que las mujeres reportan más distrés psicológico que los hombres - y socioculturales -estereotipos de los roles. Sin embargo, otros trabajos ${ }^{35}$ plantearon que estas diferencias pueden deberse a sesgos de los profesionales que diagnostican, lo cual cuestionaría - y plantearía la necesidad de revisar - estas diferencias en la prevalencia.

Asimismo, la integración de la FM junto con otras enfermedades "reumáticas" - entre las que se incluyen la artritis, la artritis reumatoidea, lupus y esclerosis múltiple - pareciera responder no tanto al hecho de sus semejanzas sintomáticas, sino al hecho de que su conceptualización como entidad mórbida parte de la reumatología como especialidad médica. En efecto, los criterios diagnósticos corresponden al $A C R^{1}$, y la enfermedad como tal está inserta dentro del grupo de los "Trastornos de los tejidos blandos de las Enfermedades del sistema músculo-esquelético y tejido conectivo" ${ }^{4}$. Este hecho explica, a su vez, que los especialistas más consultados en los artículos sean reumatólogos. Sin embargo, es menester mencionar que la mayoría de los especialistas mencionados corresponden a las disciplinas biomédicas, y que incluyen además a neurólogos, psiquiatras, urólogos, genetistas, psicólogos, lo cual refleja el carácter multidimensional de la FM². De hecho, es común encontrar también que se incluya a la FM dentro de los llamados trastornos somáticos ${ }^{36}$, correspondientes a las nosografías psicológico-psiquiátricas actuales, aunque no hay mención sobre esto en los artículos analizados.

En cuanto a las ofertas terapéuticas, abordamos este aspecto desde dos perspectivas diferentes. Si nos circunscribimos al "tema" de los artículos en los que se menciona la FM, encontramos que la 
mayoría se corresponde con terapias o actividades ligadas al campo de las terapias alternativas o New $\mathrm{Age}^{37}$, en especial tai-chi, meditación o acupuntura. Esto significa que en los artículos en los que se abordan estos temas, la FM aparece como un target de aplicación o utilización de este tipo de terapias o actividades. Esto es coherente con las tendencias actuales de difusión de las terapias alternativas para diferentes aplicaciones, tanto de enfermedades biomédicas como de otra índole, y en diferentes contextos ${ }^{38,39}$, como así también con las corrientes que investigan la aplicación o el uso de terapias alternativas/complementarias para la FM ${ }^{40-42}$.

Sin embargo, si nos limitamos a las terapias asociadas específicamente con la FM, las opciones terapéuticas se vinculan más con los tratamientos biomédicos, entre los que se incluyen fármacos antidepresivos, anticonvulsivos y analgésicos, abordajes psicoterapéuticos o educativos, kinesiología y, en menor medida, terapias alternativas o no convencionales. En la mayoría de los artículos se prioriza su uso combinado. Esto coincide, asimismo, con el estado del arte en materia científica sobre la FM, en el que encontramos que las ofertas son variadas y que las recomendaciones se orientan hacia los tratamientos multicomponentes ${ }^{43,44}$. De todas maneras, no existe un criterio homogéneo entre los distintos artículos que abordan esta temática, lo cual reafirma, asimismo, el carácter ambiguo y controvertido de esta afección, en especial en lo que hace a sus hipótesis causales y su complejidad sintomática.

También es menester destacar que ningún artículo hace referencia a la eficacia de las terapias mencionadas. Únicamente es posible rastrear que algunos recursos medicamentosos - como los analgésicos - no tienen efectos sobre el dolor percibido de los padecientes, o bien la aparición de fármacos específicos, como la Pregabalina, sobre la cual se discute respecto de su utilidad. Sin embargo, pese a que en muchos casos se citan estudios o se presenta el testimonio de especialistas en el tema, no hay alusión de los estudios científicos que apoyen o no claramente la utilización de determinadas estrategias terapéuticas. Esto también nos lleva a pensar en el lugar de la psicología, dado que, pese a la existencia de terapias específicas para el tratamiento del dolor crónico, incluyendo la $\mathrm{FM}^{10,44}$, que presentan estudios que apoyan su eficacia y con mínimos efectos secundarios, en ningún momento han sido tenidos en cuenta por estas publicaciones.

En síntesis, la información analizada en los artículos recabados nos permite registrar de qué manera se difunde la información sobre la FM en la población general. Como habíamos señalado, esto es de fundamental interés dado el impacto que tienen los medios de comunicación en las creencias y las actitudes de las personas respecto de los temas de salud y ciencia ${ }^{16}$. En lo que respecta a la FM, la información que circula es en general difusa, lo cual puede deberse, en sí, al carácter inespecífico que presenta la FM en la literatura especializada. Sin embargo, hay pocas referencias respecto del estado del arte y de los avances en materia científica respecto de su constitución como entidad mórbida como de las alternativas de acción de quienes se encuentran con este diagnóstico. Esto se acentúa aún más cuando intentamos rastrear datos del contexto local -Argentina o Latinoamérica-, dado que la mayoría de la información se limita a contextos foráneos.

En cuanto al lugar de la psicología, encontramos que la información que se brinda es limitada. Pese a que existen referencias del impacto de las variables psicológicas en la FM y en la percepción del dolor ${ }^{6}$, la psicología aparece como una disciplina homogénea, sin referencia a especialidades, aplicaciones o corrientes teóricas, las cuales sí son referidas en la mayoría de los artículos científicos que vinculan a la FM y psicología. Tampoco aparecen referencias respecto de aplicaciones específicas de tratamientos psicológicos para este tipo de dolencia.

El análisis temático realizado también nos permitió dar cuenta de temas que esperábamos que fueran abordados en los artículos, pero que no aparecieron, tales como: la eficacia o apoyo empírico de los tratamientos, referencias respecto de la adherencia al tratamiento, efectos secundarios de los fármacos, entre otros. De todas maneras, rescatamos que, pese a que muchas veces la información es incompleta o carente de referencias a la literatura especializada, ésta no se contradice con el estado del arte actual y, en definitiva, ubica a la FM dentro de la agenda de temas que circulan en los medios masivos. Sin embargo, consideramos que el lugar de la psicología se presenta aún limitado, por lo que es menester continuar promoviendo los avances en materia científica para su difusión en la población general. 


\section{Referencias}

1. Wolfe F, Smythe HA, Yunus MB, Bennett RM, Bombardier C, Goldenberg DL, et al. The American College of Rheumatology 1990 criteria for the classification of fibromyalgia: report of the Multicenter Criteria Committee. Arthritis Rheum. 1990; 33(2):160-72.

2. Wolfe F, Clauw DJ, Fitzcharles MA, Goldenberg DL, Katz RS, Mease P, et al. The American College of Rheumatology preliminary diagnostic criteria for fibromyalgia and measurement of symptom severity. Arthritis Care Res. 2010; 62(5):600-10.

3. Wolfe F. Stop using the American College of Rheumatology criteria in the clinic. J Rheumatol. 2003; 30(8):1671.

4. World Health Organization. The ICD-10 classification of mental and behavioural disorders: clinical descriptions and diagnostic guidelines. Geneva: WHO; 1992.

5. Inanici F, Yunus MB. History of fibromyalgia: past to present. Current Pain Headache Rep. 2004; 8(5):369-78.

6. Melzack R, Wall PD. Pain mechanisms: a new theory. Surv Anesthesiol. 1967; 11(2):89-90.

7. Griffies WS. Believing in the patient's capacity to know his mind: a psychoanalytic case study of fibromyalgia. Psychoanal Inquir. 2010; 30(5):390-404.

8. Suhr JA. Neuropsychological impairment in fibromyalgia: relation to depression, fatigue, and pain. J Psychosom Res. 2003; 55(4):321-9.

9. Otis JD. Managing chronic pain: a cognitive-behavioral therapy approach workbook. Oxdord: Oxford University Press; 2007.

10. Thorn BE. Cognitive therapy for chronic pain: a step-by-step guide. New York: Guilford Press; 2004.

11. Fordyce WE, Fowler RS, DeLateur B. An application of behavior modification technique to a problem of chronic pain. Behav Res Ther. 1968; 6(1):105-7.

12. Schudson M. The Sociology of news. New York: W.W. Norton \& Company; 2003.

13. Rodrigues AD. Experiência, modernidade e campo dos media. Lisboa: Universidade Nova de Lisboa; 1999 [acesso 2015 Jun 2]. Disponível em: http://www.bocc.ubi.pt/_esp/ autor.php?codautor $=2$.

14. Dimopoulos K, Koulaidis V. The socio-epistemic constitution of science and technology in the Greek press: an analysis of its presentation. Public Understand Sci. 2002; 11(3):225-41.

15. Nelkin D. Selling science: how the press covers science and technology. New York: Freeman; 1995.

16. Pellechia MG. Trends in science coverage: a content analysis of three US newspapers. Public Understand Sci. 1997; 6(1):49-68.

17. Moscovici S. El psicoanálisis, su imagen y su público. Buenos Aires: Huemul; 1979.

18. Hagger MS, Orbell S. A meta-analytic review of the common-sense model of illness representations. Psychol Health. 2003; 18(2):141-84.

19. Karasz A, Dempsey K. Health seeking for ambiguous symptoms in two cultural groups: a comparative study. Transcult Psychiatr. 2008; 45(3):415-38.

20. Kleinman AM. Patients and healers in the context of culture. Berkeley: University of California Press; 1980.

21. Pierret J. The illness experience: state of knowledge and perspectives for research. Soc Health Illness. 2003; 25(3):4-22. 
22. Souza RA, Brandão ER. À sombra do aborto: o debate social sobre a anticoncepção de emergência na mídia impressa brasileira (2005-2009). Interface (Botucatu). 2012; 16(40):161-75.

23. Rios C, Ortega F, Zorzanelli R, Nascimento LF. Da invisibilidade à epidemia: a construção narrativa do autismo na mídia impressa brasileira. Interface (Botucatu). 2015; 19(53):325-35.

24. Brigeiro M, Maksud I. Aparição do viagra na cena. Estud Fem. 2009; 17(1):71-88.

25. Braun V, Clarke V. Using thematic analysis in psychology. Qual Res Psychol. 2006; 3(2):77-101.

26. Freidin B, Ballesteros $M$. La difusión transnacional de medicinas alternativas: la presencia del Ayurveda en la prensa argentina. Pap Trabajo. 2012; 6(10):128-59.

27. Kornblit AL, Jones D, Pérez G, Verardi M. El SIDA en la prensa escrita Argentina. Buenos Aires: Facultad de Ciencias Sociales, Universidad de Buenos Aires; 2001. (Documentos de Trabajo, 25).

28. Poblet V. Cansancio y malestar, cómo combatir la fibromalgia [Internet]. Rev Susana 8 May 2012; Su Bienestar [acesso 2014 Jul 10]. (8). Disponível em: http://www. revistasusana.com/1470483-fibromalgia-cansancio-y-malestar-generalizado-comocombatirla

29. Luna P. El dolor también tiene sexo [Internet]. El Pais. 2008 Nov 4 [acesso 2014 Jul 10]. Disponível em: http://www.pagina12.com.ar/diario/sociedad/3-114932-2008-11-13. html.

30. Román V. Afirman que ciertos alimentos influyen en el estado de ánimo [Internet]. Clarín. 28 Jul 2006 [acesso 2014 Jul 10]. Disponível em: http://old.clarin.com/ diario/2006/07/28/sociedad/s-03401.htm

31. Crofford LJ, Clauw DJ. Fibromyalgia: where are we a decade after the American College of Rheumatology classification criteria were developed? [editorial]. Arthritis Rheum. 2002; 46(5):1136-8.

32. Mease P. Fibromyalgia syndrome: review of clinical presentation, pathogenesis, outcome measures, and treatment. J Rheumatol. 2005; 75 Supl:6-21.

33. Ponciano Aragón LE. Fibromialgia: perspectivas de um campo problemático. Interface (Botucatu). 2010; 14(32):166-9.

34. Yunus MB. The role of gender in fibromyalgia syndrome. Curr Rheumatol Rep. 2001; 3(2):128-34.

35. Katz JD, Mamyrova G, Guzhva O, Furmark L. Gender bias in diagnosing fibromyalgia. Gend Med. 2010; 7(1):19-27.

36. American Psychiatric Association. The diagnostic and statistical manual of mental disorders: DSM 5. Washington (DC): American Psychiatric Association Press; 2013.

37. Carozzi MJ. Nueva era y terapias alternativas: construyendo significados en el discurso y la interacción. Buenos Aires: UCA; 2001.

38. Vincent C, Furnham A. Why do patients turn to complementary medicine? An empirical study. Br J Clin Psychol. 1996; 35(1):37-48.

39. Saizar MM, Sarudiansky M, Korman GP. Mental health and new therapeutic complementarities: the experience in two public hospitals in Buenos Aires, Argentina. Psicol Soc. 2013; 25(2):451-60.

40. Lind BK, Lafferty WE, Tyree PT, Diehr PK, Grembowski DE. Use of complementary and alternative medicine providers by fibromyalgia patients under insurance coverage. Arthritis Care Res. 2007; 57(1):71-6. 
41. Sarac AJ, Gur A. Complementary and alternative medical therapies in fibromyalgia. Curr Pharmacol Des. 2006; 12(1):47-57.

42. Terhorst L, Schneider MJ, Kim KH, Goozdich LM, Stilley CS. Complementary and alternative medicine in the treatment of pain in fibromyalgia: a systematic review of randomized controlled trials. J Manipulative Physiol Ther. 2011; 34(7):483-96.

43. Fitzcharles MA, Ste-Marie PA, Goldemberg DL, Pereira JX, Abbey S, Choinière $M$, et al. 2012 Canadian guidelines for the diagnosis and management of fibromyalgia syndrome: executive summary. Pain Res Manag. 2013; 18(3):119.

44. Flor H, Turk D. Chronic pain: an integrated biobehavioral approach. Seattle: IASP Press; 2012.

Sarudiansky M. A análise temática da fibromialgia na mídia impressa argentina: descrições gerais e o papel da psicologia. Interface (Botucatu). 2016; 20(56):25-36.

Desde sua conceitualização em 1990, a Fibromialgia (FM) vem sendo discutida em termos de sua validade como diagnóstico, sua definição como doença e seus tratamentos. Estas questões têm sido abordadas por diferentes disciplinas biomédicas e sociais, entre as quais a psicologia, a psiquiatria, a antropologia e a sociologia. Neste artigo discutimos como se difundem os saberes a respeito da FM na Argentina, a partir de uma análise temática dos artigos publicados sobre esta síndrome na mídia impressa. Analisaremos a forma como as peças jornalísticas descrevem a FM - suas características gerais, hipóteses causais e tratamentos - e como a mídia aborda o papel da psicologia em seu tratamento.

Palavras-chave: Fibromialgia. Meios de Comunicação. Análise temática. Psicologia.

Recebido em 12/01/15. Aprovado em 07/07/15. 